\title{
Observation of new neutron-rich isotopes in the vicinity of ${ }^{110} \mathrm{Zr}$
}

T. Sumikama $\odot,{ }^{1,2,{ }^{*}}$ N. Fukuda, ${ }^{1}$ N. Inabe, ${ }^{1}$ D. Kameda, ${ }^{1}$ T. Kubo, ${ }^{1}$ Y. Shimizu,,${ }^{1}$ H. Suzuki, ${ }^{1}$ H. Takeda, ${ }^{1}$ K. Yoshida, ${ }^{1}$ H. Baba, ${ }_{1}^{1}$ F. Browne, ${ }^{3,1}$ A. M. Bruce, ${ }^{3}$ R. Carroll, ${ }^{4}$ N. Chiga,${ }^{2,1}$ R. Daido, ${ }^{5}$ F. Didierjean, ${ }^{6}$ P. Doornenbal,,${ }^{1}$ Y. Fang, ${ }^{5}$ G. Gey, ${ }^{7,1,8}$ E. Ideguchi, ${ }^{9}$ T. Isobe, ${ }^{1}$ S. Lalkovski, ${ }^{10}$ Z. Li,${ }^{11}$ G. Lorusso, ${ }^{1,12}$ R. Lozeva,,${ }^{6,13}$ H. Nishibata, ${ }^{5}$ S. Nishimura, ${ }^{1}$ I. Nishizuka, ${ }^{2}$ A. Odahara, ${ }^{5}$ Z. Patel, ${ }^{4}$ Zs. Podolyák, ${ }^{4}$ P. H. Regan, ${ }^{4,12}$ S. Rice, ${ }^{4}$ O. J. Roberts, ${ }^{3}$ H. Sakurai, $,{ }^{1}, 14$ G. S. Simpson, ${ }^{15}$ L. Sinclair, ${ }^{16}$ P.-A. Söderström, ${ }^{1}$ M. Tanaka, ${ }^{9}$ J. Taprogge, ${ }^{17,18,1}$ H. Watanabe, ${ }^{19,1}$ V. Werner, ${ }^{20,21}$ O. Wieland, ${ }^{22}$ J. Wu, ${ }^{11,1} \mathrm{Z}$. Y. Xu, ${ }^{14}$ and A. Yagi ${ }^{5}$

${ }^{1}$ RIKEN Nishina Center, 2-1 Hirosawa, Wako, Saitama 351-0198, Japan

${ }^{2}$ Department of Physics, Tohoku University, 6-3 Aramaki-Aoba, Aoba, Sendai 980-8578, Japan

${ }^{3}$ School of Computing, Engineering and Mathematics, University of Brighton, Brighton BN2 4GJ, United Kingdom

${ }^{4}$ Department of Physics, University of Surrey, Guildford GU2 7XH, United Kingdom

${ }^{5}$ Department of Physics, Osaka University, 1-1 Machikaneyama, Toyonaka, Osaka 560-0043, Japan

${ }^{6} I P H C, C N R S / I N 2 P 3$, Université de Strasbourg, 67037 Strasbourg, France

${ }^{7}$ LPSC, Université Joseph Fourier Grenoble 1, CNRS/IN2P3, Institut National Polytechnique de Grenoble, F-38026 Grenoble Cedex, France ${ }^{8}$ ILL, 38042 Grenoble Cedex, France

${ }^{9}$ RCNP, Osaka University, Ibaraki, Osaka 567-0047, Japan

${ }^{10}$ Department of Physics, University of Sofia, 1164 Sofia, Bulgaria

${ }^{11}$ Department of Physics, Peking University, Beijing 100871, China

${ }^{12}$ National Physical Laboratory, Teddington, Middlesex TW11 OLW, United Kingdom

${ }^{13}$ Université Paris-Saclay, CNRS/IN2P3, IJCLab, 91405 Orsay, France

${ }^{14}$ Department of Physics, University of Tokyo, 7-3-1 Hongo, Bunkyo, Tokyo 113-0033, Japan

${ }^{15}$ LPSC, Université Grenoble-Alpes, CNRS/IN2P3, F-38026 Grenoble Cedex, France

${ }^{16}$ Department of Physics, University of York, Heslington, York YO10 5DD, United Kingdom

${ }^{17}$ Departamento de Física Teórica, Universidad Autónoma de Madrid, E-28049 Madrid, Spain

${ }^{18}$ Instituto de Estructura de la Materia, CSIC, E-28006 Madrid, Spain

${ }^{19}$ International Research Center for Nuclei and Particles in the Cosmos, Beihang University, Beijing 100191, China

${ }^{20}$ Wright Nuclear Structure Laboratory, Yale University, New Haven, Connecticut 06520-8120, USA

${ }^{21}$ Institut für Kernphysik, Technische Universität Darmstadt, 64289 Darmstadt, Germany

${ }^{22}$ INFN Sezione di Milano, I-20133 Milano, Italy

(Received 6 July 2020; accepted 13 January 2021; published 27 January 2021)

\begin{abstract}
Neutron-rich radioactive isotope (RI) beams in the vicinity of ${ }^{110} \mathrm{Zr}$ were produced using the in-flight fission of a $345-\mathrm{MeV} /$ nucleon ${ }^{238} \mathrm{U}$ beam at the RIKEN Radioactive Isotope Beam Factory. The RI beams were separated in flight by the large-acceptance two-stage fragment separator BigRIPS. Isotopes were clearly identified in a low-background particle-identification plot, which was obtained from the measurements of time of flight, magnetic rigidity, and energy loss using beam-line detectors placed in the BigRIPS separator and the ZeroDegree spectrometer. Nine new isotopes, ${ }^{101} \mathrm{Br},{ }^{102} \mathrm{Kr},{ }^{105,106} \mathrm{Rb},{ }^{108} \mathrm{Sr},{ }^{110,111} \mathrm{Y},{ }^{114} \mathrm{Zr}$, and ${ }^{117} \mathrm{Nb}$, are reported for the first time, with their measured production cross sections compared with the extrapolations of lighter isotopes down to the femtobarn regime.
\end{abstract}

DOI: 10.1103/PhysRevC.103.014614

\section{INTRODUCTION}

Neutron-rich nuclei in the vicinity of ${ }_{40}^{110} \mathrm{Zr}_{70}$ are known to exhibit substantial shape evolution as a function of proton and neutron numbers. Over the past decade, spectroscopic information of even-even neutron-rich $\mathrm{Kr}, \mathrm{Sr}$, and $\mathrm{Zr}$ nuclei has been extended toward their heaviest discovered isotopes at the mass number $A \approx 110$ [1-9]. To search for a predicted shape

*sumikama@ ribf.riken.jp transition from deformed to spherical at the neutron number $N \approx 70$ [10], the even-even $\mathrm{Zr}$ isotopes with $A=108$ and 110 were experimentally studied $[1,7]$, with the conclusion that the deformed configuration remains favored at low excitations up to $A=110$.

Recent isotope discoveries in the neutron-rich regions of the nuclear chart have allowed the experimental study of exotic nuclei close to, or even on, a predicted flow path of the rapid-neutron capture $(r)$ process of nucleosynthesis [11-17]. Measurements of $\beta$-decay half-lives of these isotopes demonstrated the importance of such experimental 
TABLE I. Summary of the horizontal-slit conditions at F1 and F2 foci in four RI-beam settings. The positive direction for the slits is the right in a view from downstream. The arrows indicate the value is the same as the preceding column. The irradiation time and dose of the primary ${ }^{238} \mathrm{U}$ beam are written for each setting.

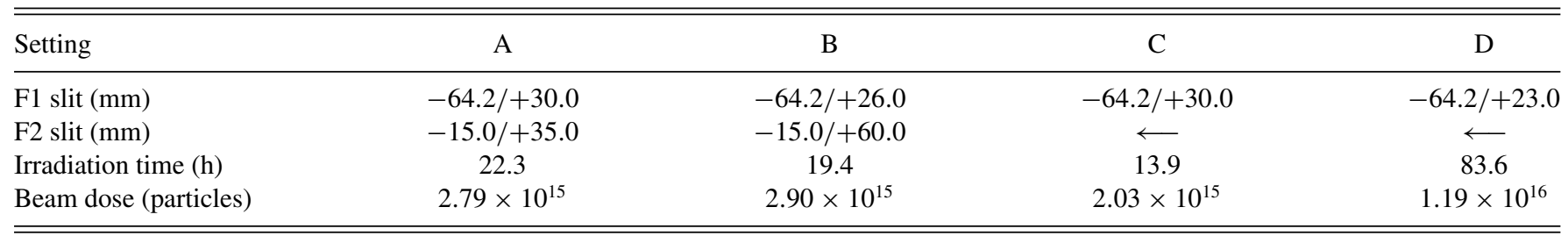

inputs to understand the $r$-process abundance in the solar system [18-21].

In the present paper, we report for the first time the measurement and identification of the most neutron-rich isotopes of the elements from bromine (atomic number $Z=35$ ) to niobium $(Z=41)$. The radionuclides of interest were produced in an experiment aimed at the study of the radioactive decay properties of neutron-rich nuclei in the $A \approx 110$ region at the RIKEN Radioactive Isotope Beam Factory (RIBF), in which the EUroball RIKEN Cluster Array (EURICA) and a fast-timing array of $\mathrm{LaBr}_{3}(\mathrm{Ce})$ detectors (FATIMA) were used for $\gamma$-ray detection $[22,23]$. Some results from this experiment were published in Refs. [5,24,25].

\section{EXPERIMENT}

Neutron-rich radioactive isotope (RI) beams were produced by the in-flight fission reaction of a $345-\mathrm{MeV} /$ nucleon ${ }^{238} \mathrm{U}$ beam on a $2.92-\mathrm{mm}$ beryllium target. The ${ }^{238} \mathrm{U}$-beam intensity was 6.3 pnA on average. The RI beams were separated in flight by the large-acceptance two-stage fragment separator BigRIPS [26,27] using two wedge-shaped aluminum degraders at momentum-dispersive foci, F1 and F5, of the first and second stages, respectively (see Fig. 1 in Ref. [27] for the layout and foci of the BigRIPS separator and the ZeroDegree spectrometer). The thicknesses of the F1 and F5 degraders were 6 and $4.5 \mathrm{~mm}$, respectively. The magnetic rigidity of the first dipole magnet after the beryllium target was set to 8.012 Tm. The magnetic rigidities of downstream dipole magnets were adjusted to transport a ${ }^{108} \mathrm{Y}^{39+}$ beam along the central trajectory. Those after the F1 and F5 degraders were set to 7.128 and 6.267 Tm, respectively. Table I summarizes four different settings of the slits at the dispersive focus F1 and the achromatic focus F2, used to change the mixture of the RI beams as required by the primary decay experiment [5,24,25]. The slit at the dispersive focus F5 was fully opened and that at the achromatic focus F7, being the last focus of the second stage, was set to $\pm 20 \mathrm{~mm}$. The RI beams were transported to the EURICA setup through the ZeroDegree spectrometer for decay studies. The separator settings were determined on the basis of detailed simulations using the LISE ${ }^{++}$code [28].

For the event-by-event particle identification (PID) of the RI beams, the time of flight (TOF) and magnetic rigidity, $B \rho$, were measured at the second stage of BigRIPS, and the energy loss, $\Delta E$, was measured at the ZeroDegree spectrometer. The TOF between two achromatic foci F3 and F7 of the second stage was measured using two $200-\mu \mathrm{m}-$ thick plastic scintillation detectors. Although the horizontal distribution of RI beams at F3 reached the edge of the 120-mm-wide plastic scintillator, only RI beams, for which the $\mathrm{F} 3$ position was within $\pm 45 \mathrm{~mm}$, were considered in the present analysis. This ensured a sufficient quantity of scintillation light reached both left and right photomultiplier tubes (PMTs) for a robust output signal. Two $B \rho \mathrm{s}$, before and after the F5 degrader, were determined by the track reconstruction from the position and angle measurements at three foci, F3, F5, and F7 [29], using the positionsensitive parallel plate avalanche counters (PPACs) [30]. A multisampling ionization chamber (MUSIC) [31] for $\Delta E$ measurements was placed at the final focus of the ZeroDegree spectrometer.

\section{RESULTS AND DISCUSSION}

The analysis employed in the present measurement is detailed in Ref. [17], which reports the discovery of new isotopes from different RI-beam settings during the EURICA campaign. The atomic number, $Z$, and mass-to-charge ratio, $A / Q$, were determined from the $B \rho$, TOF, and $\Delta E$ values as described in Ref. [29]. Figure 1 shows the PID plot using $Z$ and $A / Q$ for the isotopes transported through the BigRIPS and ZeroDegree spectrometers, which was confirmed by the isomeric tagging method, measuring $\gamma$ rays emitted from the isomeric state in ${ }^{108} \mathrm{Zr}[1,2]$. The resolutions of $Z$ and $A / Q$ were $0.4 \%$ and $0.068 \%$ in $1 \sigma$, respectively. The $A / Q$ resolu-

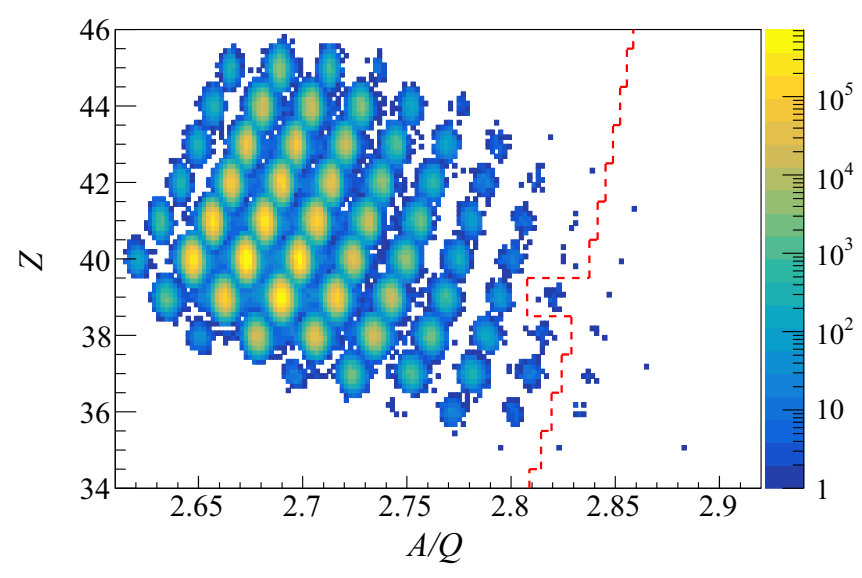

FIG. 1. Particle identification using the mass-to-charge ratio $A / Q$ and atomic number $Z$ for fission fragments produced in the reaction of ${ }^{238} \mathrm{U}$ on the beryllium target at $345 \mathrm{MeV} /$ nucleon. Previously undiscovered isotopes are to the right of the dashed red line. 

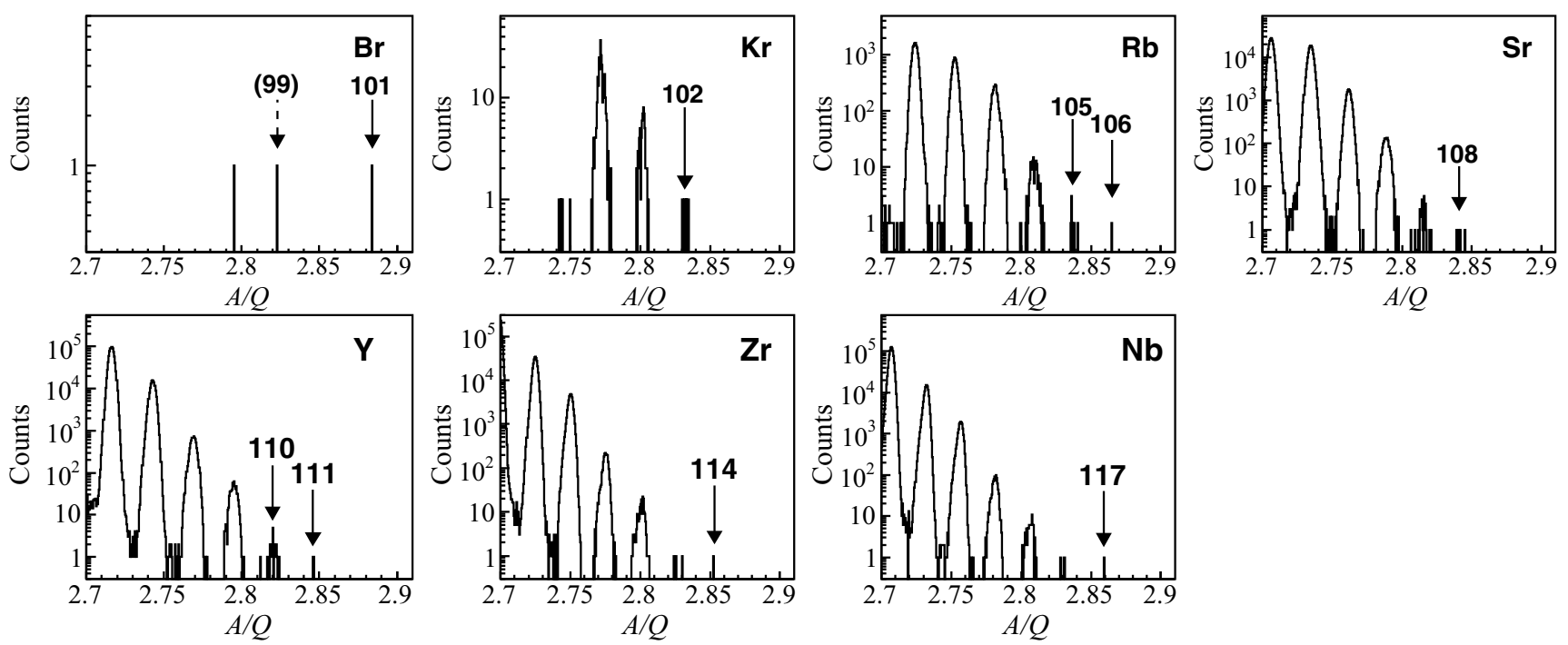

FIG. 2. $A / Q$ spectrum for each isotope with a gate of $\left|Z-Z_{0}\right|< \pm 0.3$, corresponding to $\pm \approx 2 \sigma$, where $Z_{0}$ is the integer value of the atomic number. The arrow labels indicate the mass number of new isotopes observed in the present study. One event of ${ }^{99} \mathrm{Br}$ is not claimed as a new isotope, because it was impossible to determine the production cross section.

tion was mainly caused by the TOF resolution, $\sigma_{\mathrm{TOF}}$, for the flight length of $47.0 \mathrm{~m}$, since the $A / Q$ uncertainty propagated from $\sigma_{\mathrm{TOF}}$ of $100 \mathrm{ps}$ was $0.062 \%$. The rise times of the charge signals of the PMTs were not enough fast to obtain a good $A / Q$ resolution of $0.035 \%$ achieved in the previous work [12], since the voltages applied to them were optimized not only to the present experiment but also to all experiments during the EURICA campaign.

The analysis for the background rejection was performed according to descriptions in Refs. [17,29], and only gates effectively rejecting background events, described below, were applied in the present work. Events which showed inconsistent relations among measured values related to the plastic-scintillation detectors and the $B \rho$ determination were considered as background and rejected. The atomic number was determined also using the energy loss in the F5 degrader, which was calculated from the $B \rho$ and TOF values by assuming $Q=Z$ before and after F5 and no reaction [16,17]. It was compared with the atomic number from the measured $\Delta E$ in the MUSIC to reject inconsistent events caused by the charge-state change at F5 and reaction on the detectors and F5 degrader.

The $A / Q$ spectrum for each isotopic chain is shown in Fig. 2, where the events having $Z$ within $a \approx 2 \sigma$ difference from the integer value were selected. Although events for the hydrogenlike ions $(Q=Z-1)$ before and after F5 were not rejected by the above gates, only the fully stripped ions were observed. Ten candidates for previously unreported neutronrich isotopes were produced, namely, events corresponding to fully stripped ions of ${ }^{99,101} \mathrm{Br},{ }^{102} \mathrm{Kr},{ }^{105,106} \mathrm{Rb},{ }^{108} \mathrm{Sr},{ }^{110,111} \mathrm{Y}$, ${ }^{114} \mathrm{Zr}$, and ${ }^{117} \mathrm{Nb}$. The $A / Q$ values of new-isotope events were consistent with those extrapolated from other isotopes.

For each candidate, except for ${ }^{99} \mathrm{Br}$, the production cross section, $\sigma$, was compared to systematic trends to check whether the number of measured events was reasonable and a significance test was performed to exclude the possibility that the candidate stems from the hydrogenlike ion with a lighter mass. Almost all ${ }^{99} \mathrm{Br}$ ions transmitted to $\mathrm{F} 2$ were stopped at the F2 slit; therefore, it was impossible to determine $\sigma$ and ${ }^{99} \mathrm{Br}$ is not claimed as a new isotope in the present study. The ${ }^{100} \mathrm{Br}$ ions was unobserved due to the low transmission efficiency and a production yield as low as newly observed isotopes.

The $\sigma$ values were obtained using the transmission efficiency evaluated with LISE $^{++}$(version 12.1.2) [28], as shown by filled symbols in Fig. 3. The $\sigma$ values of the new isotopes are summarized in Table II. The systematic uncertainty was common for the EURICA campaign and estimated to be $50 \%$ in Ref. [17]. The previous results obtained at the RIBF $[11,12,17]$, shown by open symbols in Fig. 3, are consistent with the present work. The $\sigma$ values of the new and known isotopes follow the same systematic trend.

Predictions by the three excitation-energy region (3EER) model implemented in LISE $^{++}$(version 8.4.1) for the abrasion fission are shown by solid lines in Fig. 3. While there are a lot of fissionable isotopes after the abrasion reaction of ${ }^{238} \mathrm{U}$ on the beryllium target, the 3EER model uses representatively three kinds of fissionable isotopes with low, mid, and high excitation energies. The parameters of fissionable isotopes given in Ref. [32] were applied. For the $\mathrm{Zr}$ and $\mathrm{Nb}$ isotopes, the 3EER model reproduces very well the measured cross sections especially for $\sigma \gtrsim 10^{-6} \mathrm{mb}$. The predictions of heavier $\mathrm{Zr}$ and $\mathrm{Nb}$ isotopes and of $\mathrm{Br}, \mathrm{Kr}, \mathrm{Sr}$, and $\mathrm{Rb}$ isotopes are underestimated.

In a model of an initial fissile nuclei (IFN) analyzer implemented in LISE ${ }^{++}$(version 12.1.2) [33], the abrasion reaction is calculated to obtain the fissionable isotopes and their excitation energies for each fission fragment. The predictions by the IFN1 model in the IFN analyzer are shown by dashed lines in Fig. 3, where a mean value is applied for the excitation energy after the abrasion reaction. Conversely, the $\sigma$ values of isotopes far from the stability line are overestimated. The 


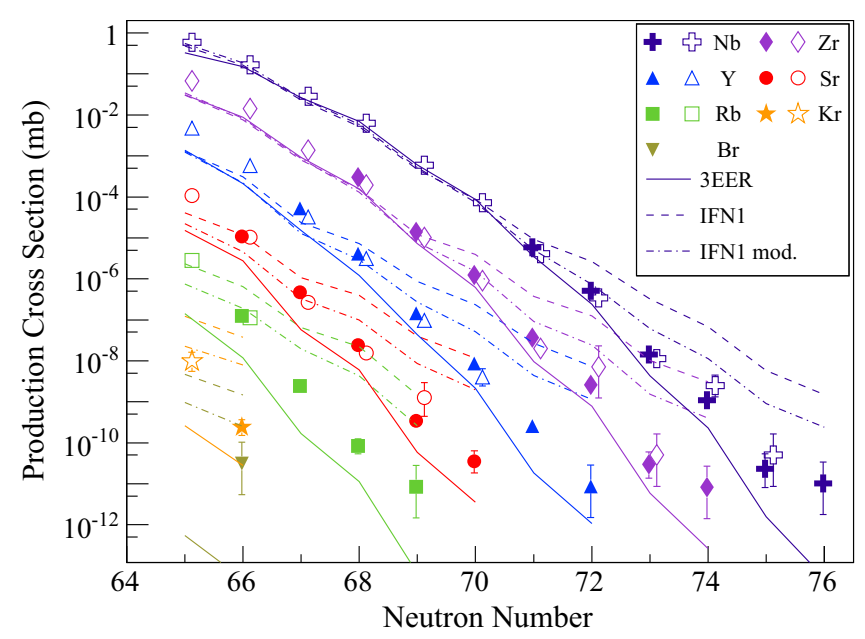

FIG. 3. Production cross sections of RI beams from the in-flight fission of $345-\mathrm{MeV} /$ nucleon ${ }^{238} \mathrm{U}$ beam on the beryllium target. The filled symbols are from the present results and the open symbols are previous results obtained at the RIBF $[11,12,17]$. The error bars show the statistical uncertainties. The solid and dashed lines are the predictions by the 3EER model with the parameters given in Ref. [32], and by the IFN1 model [33], respectively. The dash-dotted lines are the IFN1 prediction with modified parameters. The parameters for prefragment-excitation energy and its $\sigma$ were changed from 27 and 18 to 48 and 22, respectively. The $f$ parameter [34] for the fission-fragment excitation energy was changed from 0.0045 to 0.0017 .

parameters in the IFN1 model were optimized to the data shown in Fig. 3. The revised predictions shown by dash-dotted lines are still overestimated. Although further improvements of the IFN analyzer are necessary, the increase of the cross sections suggests an importance of the fragment dependence of the fissionable isotopes as mentioned in Ref. [33].

In the previous experiments $[11,12,16,17,27,29]$, the hydrogenlike ions in the RI beams were significant contaminants around new-isotope events in the PID plot. The hydrogenlike ions with $A=A_{1}+3$ and $Q=Z_{1}-1$ have a similar $A / Q$

TABLE II. Counts, production cross sections $\sigma$, and upper limits of the $p$ values for the new isotopes. The statistical uncertainties are written for $\sigma$. The significant test using the $p$ values [35] was performed for the new-isotope discovery as described in Ref. [12]. See text for the $p$-value evaluation.

\begin{tabular}{lcrr}
\hline \hline Nuclide & Counts & \multicolumn{1}{c}{$\sigma(\mathrm{mb})$} & $p$ value $(\%)$ \\
\hline${ }^{101} \mathrm{Br}$ & 1 & $\left(3.1_{-2.6}^{+7.2}\right) \times 10^{-11}$ & $<0.01$ \\
${ }^{102} \mathrm{Kr}$ & 7 & $\left(2.4_{-0.9}^{+1.3}\right) \times 10^{-10}$ & $<0.01$ \\
${ }^{105} \mathrm{Rb}$ & 8 & $\left(8.3_{-2.9}^{+4.1}\right) \times 10^{-11}$ & $<0.01$ \\
${ }^{106} \mathrm{Rb}$ & 1 & $\left(8_{-7}^{+19}\right) \times 10^{-12}$ & 0.03 \\
${ }^{108} \mathrm{Sr}$ & 4 & $\left(3.5_{-1.7}^{+2.8}\right) \times 10^{-11}$ & $<0.01$ \\
${ }^{110} \mathrm{Y}$ & 25 & $(2.5 \pm 0.5) \times 10^{-10}$ & $<0.01$ \\
${ }^{111} \mathrm{Y}$ & 1 & $\left(9_{-7}^{+20}\right) \times 10^{-12}$ & 0.05 \\
${ }^{114} \mathrm{Zr}$ & 1 & $\left(8_{-7}^{+19}\right) \times 10^{-12}$ & $<0.01$ \\
${ }^{117} \mathrm{Nb}$ & 1 & $\left(1.0_{-0.8}^{+2.4}\right) \times 10^{-11}$ & $<0.01$ \\
\hline \hline
\end{tabular}

value to the fully stripped ions with $A=A_{1}$ and $Q=Z_{1}$. For instance, the $A / Q$ difference between ${ }_{39}^{108} \mathrm{Y}^{38+}$ and ${ }_{39}^{111} \mathrm{Y}^{39+}$ is $0.14 \%$ in relative terms, which is twice as large as the experimental $A / Q$ resolution of $0.07 \%$. The hydrogenlike ions were not observed in the present PID plot. Nevertheless, the hydrogenlike ions are still potential origins of misidentification for the new-isotope events. The significance test using $p$ values [35], which are the probability misidentifying all of the measured events, was performed as described in Ref. [12]. As an example, the $p$-value estimation for the new isotope, ${ }^{111} \mathrm{Y}$, is described. For an unobserved ${ }^{108} \mathrm{Y}^{38+}$, it is necessary to estimate the width of the $A / Q$ distribution and the upper limit of the yield. The $A / Q$ width of ${ }^{108} \mathrm{Y}^{38+}$ was assumed to be the same as one of the observed ${ }^{108} \mathrm{Y}^{39+}$ events. The upper limit of the ratio of the hydrogenlike to fully stripped ions was evaluated by using ${ }_{42}^{113}$ Mo events instead of the new isotopes, since the fraction of the hydrogenlike ion for the new isotopes with $Z<42$ is expected to become smaller than that of ${ }^{113} \mathrm{Mo}$. The upper limit of the fraction of the hydrogen-like ions of ${ }^{113} \mathrm{Mo}$ was determined to be $1.3 \times 10^{-6}$ from the ratio of 1.1 hydrogenlike events, being the upper limit of the $68 \%$ confidence interval for nonobservation of ${ }_{42}^{113} \mathrm{Mo}^{41+}$, to $8.55 \times 10^{5}$ events for ${ }_{42}^{113} \mathrm{Mo}^{42+}$. The expected upper limit of the ${ }^{108} \mathrm{Y}^{38+}$ yield was given as $1.0 \times 10^{-2}$ by the product of the ${ }^{108} \mathrm{Y}^{39+}$ yield, 7909, and the upper limit of the fraction of the hydrogenlike ion. Using the $A / Q$ width and yield upper limit expected for ${ }^{108} \mathrm{Y}^{38+}$, the $p$ value for the ${ }^{111} \mathrm{Y}$ event was evaluated to be $0.05 \%$. The $p$ values for all the new isotopes are summarized in Table II. The $p$ values less than $1 \%$ and the systematic trend of $\sigma$ provide the evidence for the first observations of ${ }^{101} \mathrm{Br},{ }^{102} \mathrm{Kr},{ }^{105,106} \mathrm{Rb},{ }^{108} \mathrm{Sr},{ }^{110,111} \mathrm{Y},{ }^{114} \mathrm{Zr}$, and ${ }^{117} \mathrm{Nb}$.

\section{SUMMARY}

Neutron-rich RI beams were produced using a 345$\mathrm{MeV} /$ nucleon ${ }^{238} \mathrm{U}$ beam at the RIKEN RIBF and separated by the BigRIPS fragment separator. Previously unreported neutron-rich isotopes were identified from a well-resolved PID plot obtained from $B \rho$, TOF, and $\Delta E$ measurements. Nine new isotopes, ${ }^{101} \mathrm{Br},{ }^{102} \mathrm{Kr},{ }^{105,106} \mathrm{Rb},{ }^{108} \mathrm{Sr},{ }^{110,111} \mathrm{Y}$, ${ }^{114} \mathrm{Zr}$, and ${ }^{117} \mathrm{Nb}$, were discovered based on their production cross sections being consistent with systematics and significance tests using $p$ values showing a vanishing probability of misidentification.

\section{ACKNOWLEDGMENTS}

This experiment was carried out at the RIBF operated by the RIKEN Nishina Center, and the Center for Nuclear Study, the University of Tokyo. The authors acknowledge the RIBF accelerator staffs for their efforts in delivering an intense ${ }^{238} \mathrm{U}$ beam. This work was supported by JSPS KAKENHI Grants No. 2301752, No. 24740188, No. 25247045, No. 26800117, and No. 16K17680, NRF Grant No. 2016K1A3A7A09005575, STFC Grants No. ST/J000132/1, No. ST/J000051/1, No. ST/K502431/1, No. ST/L005743/1, No. ST/P003885/1, No. ST/P003982/1, and No. ST/P005314/1, DOE Grant No. DE-FG02-91ER- 
40609, BMBF Grant No. 05P19RDFN1, and Spanish Ministerio de Ciencia e Innovación under Contracts No. FPA200913377-C02 and No. FPA2011-29854-C04. P.H.R. and G.L. acknowledge additional support from the UK Department for
Business, Energy and Industrial Strategy (BEIS) via funding for the UK National Measurement System. P.-A.S. was financed by the RIKEN Foreign Postdoctoral Researcher Program.
[1] T. Sumikama et al., Phys. Rev. Lett. 106, 202501 (2011).

[2] D. Kameda et al., Phys. Rev. C 86, 054319 (2012).

[3] M. Albers et al., Phys. Rev. Lett. 108, 062701 (2012).

[4] A. Navin et al., Phys. Lett. B 728, 136 (2014).

[5] F. Browne et al., Phys. Lett. B 750, 448 (2015).

[6] Z. M. Wang et al., Phys. Rev. C 93, 054301 (2016).

[7] N. Paul et al., Phys. Rev. Lett. 118, 032501 (2017).

[8] J. Dudouet et al., Phys. Rev. Lett. 118, 162501 (2017).

[9] F. Flavigny et al., Phys. Rev. Lett. 118, 242501 (2017).

[10] M. Bender, K. Bennaceur, T. Duguet, P. H. Heenen, T. Lesinski, and J. Meyer, Phys. Rev. C 80, 064302 (2009).

[11] T. Ohnishi et al., J. Phys. Soc. Jpn. 77, 083201 (2008).

[12] T. Ohnishi et al., J. Phys. Soc. Jpn. 79, 073201 (2010).

[13] H. Alvarez-Pol, J. Benlliure, E. Casarejos, L. Audouin, D. Cortina-Gil, T. Enqvist, B. Fernández-Domínguez, A. R. Junghans, B. Jurado, P. Napolitani, J. Pereira, F. Rejmund, K.-H. Schmidt, and O. Yordanov, Phys. Rev. C 82, 041602(R) (2010).

[14] J. Kurcewicz et al., Phys. Lett. B 717, 371 (2012).

[15] T. Sumikama et al., Phys. Rev. C 95, 051601(R) (2017).

[16] N. Fukuda et al., J. Phys. Soc. Jpn. 87, 014202 (2018).

[17] Y. Shimizu et al., J. Phys. Soc. Jpn. 87, 014203 (2018).

[18] S. Nishimura et al., Phys. Rev. Lett. 106, 052502 (2011).

[19] G. Lorusso et al., Phys. Rev. Lett. 114, 192501 (2015).

[20] J. Wu et al., Phys. Rev. Lett. 118, 072701 (2017).

[21] J. Wu et al., Phys. Rev. C 101, 042801(R) (2020).
[22] P.-A. Söderström et al., Nucl. Instrum. Methods B 317, 649 (2013).

[23] M. Rudigier et al., Nucl. Instrum. Methods A 969, 163967 (2020).

[24] F. Browne et al., Phys. Rev. C 96, 024309 (2017).

[25] J. Ha et al., Phys. Rev. C 101, 044311 (2020).

[26] T. Kubo, Nucl. Instrum. Methods B 204, 97 (2003).

[27] T. Kubo, D. Kameda, H. Suzuki, N. Fukuda, H. Takeda, Y. Yanagisawa, M. Ohtake, K. Kusaka, K. Yoshida, N. Inabe, T. Ohnishi, A. Yoshida, K. Tanaka, and Y. Mizoi, Prog. Theor. Exp. Phys. 2012, 03 C003 (2012).

[28] O. Tarasov and D. Bazin, Nucl. Instrum. Methods B 266, 4657 (2008), http://groups.nscl.msu.edu/lise/lise.html.

[29] N. Fukuda, T. Kubo, T. Ohnishi, N. Inabe, H. Takeda, D. Kameda, and H. Suzuki, Nucl. Instrum. Methods B 317, 323 (2013).

[30] H. Kumagai, A. Ozawa, N. Fukuda, K. Sümmerer, and I. Tanihata, Nucl. Instrum. Methods A 470, 562 (2001).

[31] K. Kimura, T. Izumikawa, R. Koyama, T. Ohnishi, T. Ohtsubo, A. Ozawa, W. Shinozaki, T. Suzuki, M. Takahashi, I. Tanihata, T. Yamaguchi, and Y. Yamaguchi, Nucl. Instrum. Methods A 538, 608 (2005).

[32] H. Suzuki et al., Nucl. Instrum. Methods B 317, 756 (2013).

[33] http://lise.nscl.msu.edu/10_1/11_0_28_IFN_search.pdf.

[34] H. R. Faust, Eur. Phys. J. A 14, 459 (2002).

[35] P. A. Zyla et al. (Particle Data Group), Prog. Theor. Exp. Phys. 2020, 083 C01 (2020), see the section on statistics. 